\title{
Rare Spontaneous Development of Nasal Septal Abscess in End-stage Kidney Disease
}

\author{
Paolo Nikolai H. So, MD, ${ }^{1}$ Jan Alexeis C. Lacuata, MD² and Rey Jaime M. Tan, MD ${ }^{1}$ \\ ${ }^{1}$ Division of Nephrology, Department of Medicine, Philippine General Hospital, University of the Philippines Manila \\ ${ }^{2}$ Department of Otorbinolaryngology, Philippine General Hospital, University of the Philippines Manila
}

\begin{abstract}
The spontaneous development of a nasal septal abscess in patients with chronic kidney disease is hardly described in the literature. A 58-year-old man with long-standing type 2 diabetes mellitus and a history of rectal adenocarcinoma was admitted for resection of tumor recurrence. He was initiated on hemodialysis post-operatively due to worsening kidney function. He was discharged on thrice-weekly dialysis but was readmitted two months after for progressive shortness of breath. Further examination revealed severe nasal congestion from a nasal septal abscess which prompted mouth-breathing. Incision and drainage and anterior nasal packing were done, and the patient was discharged improved on broad-spectrum oral antibiotics. This case report highlights the possibility of developing nontraumatic nasal infections in patients with chronic kidney disease due to compromised host defenses.
\end{abstract}

Keywords: nasal septal abscess, chronic kidney disease, hemodialysis, diabetes mellitus

\section{INTRODUCTION}

Patients with chronic kidney disease $(\mathrm{CKD})$ are at an increased risk of developing nasal infections from compromised host defenses. ${ }^{1}$ However, a systematic search using PubMed, Cochrane Library, HerdIn, and Google Scholar revealed no published literature describing the spontaneous development of nasal septal abscesses in this patient population.

We report a rare case of nasal septal abscess presenting with persistent dyspnea in a CKD patient on adequate maintenance hemodialysis, necessitating incision and drainage as well as concomitant administration of antibiotics. This article also aims to do a brief review of the literature on the topic.

\section{CLINICAL CASE}

A 58-year-old man with type 2 diabetes mellitus for 20 years maintained on isophane insulin and with an 80 pack-year-smoking history was diagnosed to have rectal

Poster presented as part of a case series entitled "Respiratory Causes of Persistent Dyspnea Despite Hemodialysis: A Case Series" in the $18^{\text {th }}$ Asian Pacific Congress of Nephrology (Virtual) on October 2-4, 2020, at Hong Kong.

Corresponding author: Paolo Nikolai H. So, MD

Division of Nephrology

Department of Medicine

Philippine General Hospital

University of the Philippines Manila

Taft Avenue, Ermita, Manila 1000, Philippines

Email: paolonikolaiso@gmail.com adenocarcinoma. He underwent mini-laparotomy and sigmoid loop colostomy in 2017; long-course chemoradiation in 2018; systemic chemotherapy in 2019; then eventually low anterior resection, en bloc cystoprostatectomy, and cholecystectomy for tumor recurrence. He was initiated on hemodialysis post-operatively due to his worsening kidney function and was later discharged on thrice-weekly dialysis. After two months of dialysis, the patient consulted at our hospital for progressive shortness of breath. He was clinically dry, without any neck vein engorgement, 
inspiratory crackle, or edema. He was initially borderline hypotensive during his hemodialysis session, which improved with a reduction in his ultrafiltrate volume. It was observed that he had severe nasal congestion, which prompted mouthbreathing. On physical examination, a nasal septal abscess was noted (Figure 1). No history of nasal manipulation or trauma was elicited.

Aspiration was done, revealing 3 cc of purulent discharge, and samples were sent for culture and sensitivity studies. Gram staining of the nasal abscess aspirate showed $>25$ polymorphonuclear leukocytes per oil-immersion field, but no organism was seen. Culture studies were likewise negative. Unfortunately, the samples were not sent for anaerobic or acid-fast bacillus studies. The patient was initiated on Piperacillin-tazobactam $4.5 \mathrm{~g}$ intravenously as a loading dose, then $2.25 \mathrm{~g}$ every 8 hours.

Incision and drainage were done under local anesthesia, draining $20 \mathrm{cc}$ total of purulent discharge, which relieved symptoms. No Penrose drain was placed at the site of drainage. Sodium chloride nasal spray two puffs per nostril BID was started. Anterior nasal packing coated with Mupirocin was applied then removed after two days, with a note of minimal egress of serosanguinous to yellowish nasal discharge. The patient was eventually discharged on Amoxicillin + Clavulanic acid $500 \mathrm{mg} / 125 \mathrm{mg}$ TID for seven days, with normal capillary blood glucose on isophane insulin 20 units 30 minutes pre-breakfast and 10 units 30 minutes pre-dinner. He was advised of adequate sugar control to prevent recurrence of infection. He still had good residual kidney function, which allowed his subsequent HD sessions to have decreased frequency twice a week and reduced ultrafiltrate volume without recurrence of intradialytic hypotension.

The patient followed up one month later at the outpatient department due to recurrence of the nasal septal abscess, with a visible bulge on both nasal cavities (Figure 2). Repeat incision and drainage under local anesthesia was done, and mupirocin-impregnated gauze strips were inserted in both nostrils to prevent abscess reaccumulation. The patient was sent home on Clindamycin $600 \mathrm{mg}$ TID for seven days. He was advised to follow up after three days to remove the nasal pack but was lost to follow-up.

\section{DISCUSSION}

A nasal septal abscess is a collection of pus between the cartilaginous or bony septum and mucoperichondrium or mucoperiosteum. ${ }^{2}$ It is most commonly caused by trauma or nasal septal surgery but is also frequently seen in infections of nasal septal hematomas. ${ }^{2-5}$ Proposed mechanisms for its development include a direct extension from sinusitis, infections of the dental origin or underlying masses (e.g., hematoma, dermoid, or epidermoid); and hematogenous spread from septic thrombophlebitis of the orbits or cavernous sinuses. ${ }^{6}$

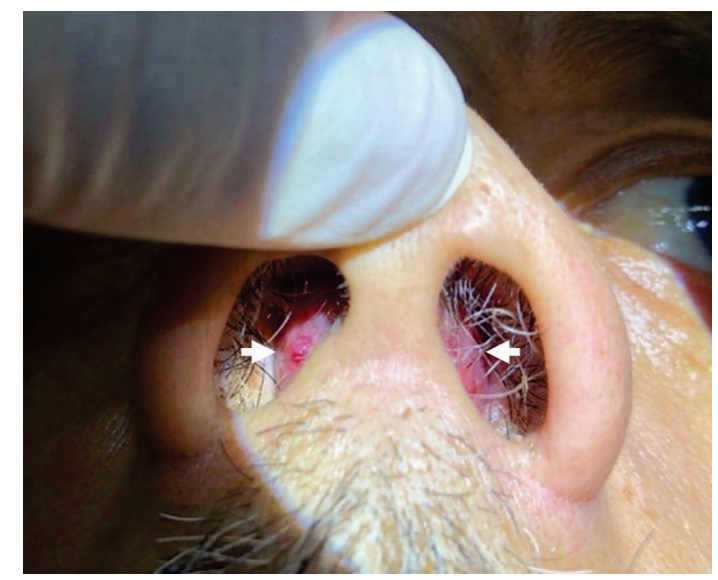

Figure 1. Bilateral pink, soft, smooth bulges (arrows) arising from the nasal septum.

Nasal septal hematomas can either resorb or be superimposed with infections, resulting in the destruction of cartilage and the clinical appearance of a saddle nose deformity. ${ }^{7}$ Kumar et al. (2004) evaluated 30 pediatric patients with renal failure undergoing peritoneal dialysis for ear, nose, and throat symptoms; and discussed that in patients who presented with epistaxis, high levels of blood urea were excreted through nasal secretions then were split into ammonia, thereby producing chemical rhinitis which might have caused congestion, nasal ulceration, and submucosal hemorrhages. ${ }^{8}$ Likewise, in our patient, it is possible that underlying submucosal bleeding may have first contributed to the development of a nasal septal hematoma, which could have been colonized by bacteria, eventually resulting in an abscess.

One case report described the development of a spontaneous, atraumatic posterior nasal septal abscess in a healthy adult. Still, most available reports on nontraumatic nasal septal abscesses involved patients with diabetes mellitus. ${ }^{9-11}$ Adler and Ritz (1980) observed spontaneous perforation of the nasal septum in 8 out of 104 patients with renal failure, documenting that such condition occurs more frequently compared to the general population. Factors identified in its pathogenesis included uremic complications, such as impairment of mucosal regeneration and the development of dry anterior rhinitis; tissue ischemia either from vascular hyperplasia secondary to hypertension or vascular calcification; and history of possible trauma to the nasal mucosa. ${ }^{12}$ At present, there is no available literature describing the development of a nasal septal abscess in patients with end-stage kidney disease without any history of nasal trauma or manipulation. Our case report documents such a rare occurrence in this patient population. Hypothetically, our patient's diminished immune function from co-morbidities of T2DM and CKD were possible contributing factors for his spontaneous nasal abscess. 

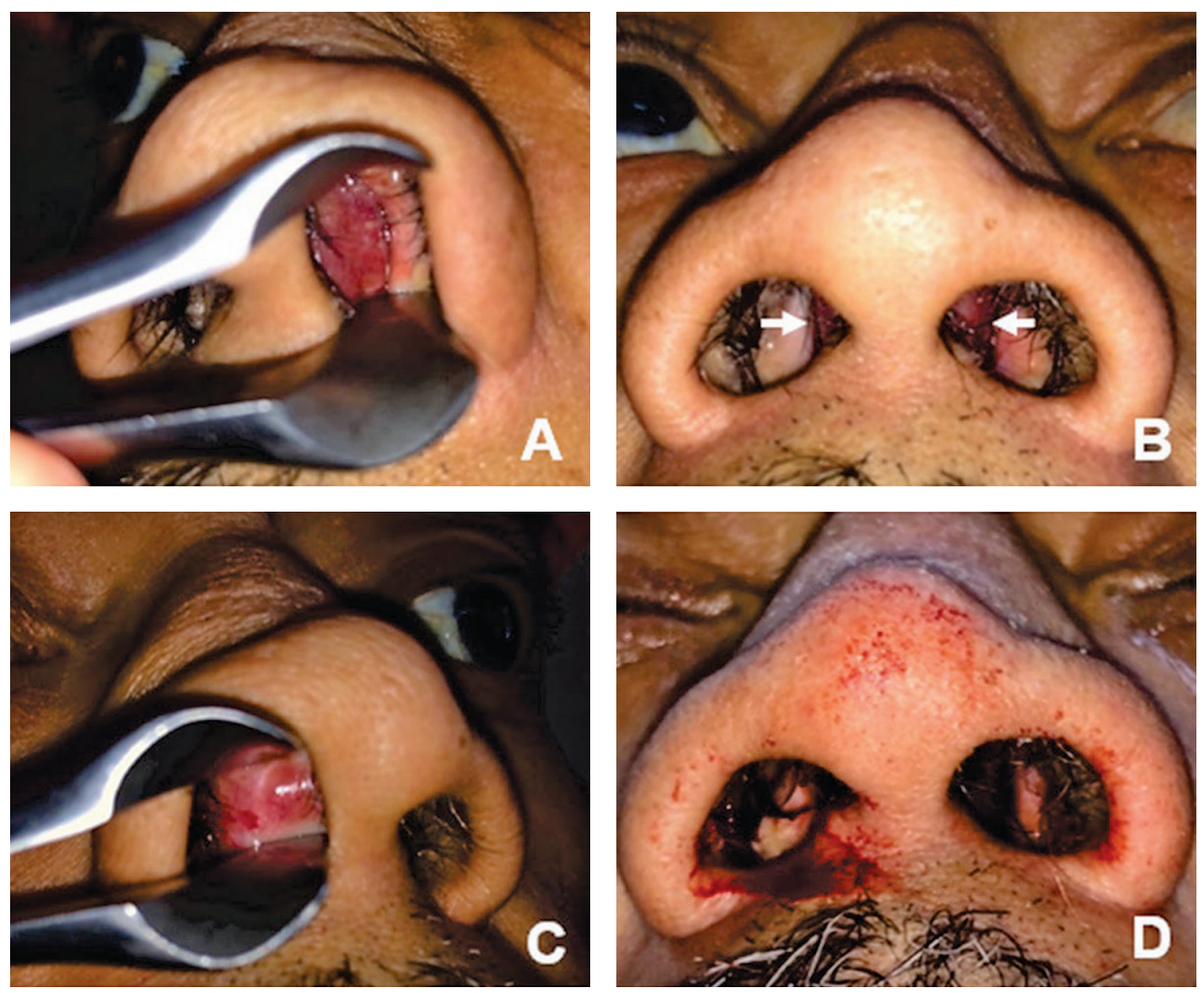

Figure 2. Recurrence of nasal septal abscess after incision and drainage one month prior. (A) Nasal septal abscess viewed from the left nostril. (B) Nasal septal abscess (arrows) viewed from below. (C) Nasal septal abscess viewed from the right nostril. (D) Repeat incision and drainage done, with the disappearance of the abscess's contour viewed from below.

A nasal septal abscess diagnosis is clinical, and patients most often present with nasal congestion or obstruction. ${ }^{4}$ Other signs and symptoms include nasal pain, fever, chills, headache, rhinorrhea, and epistaxis. ${ }^{2,13}$ Physical examination is more telling, which may reveal a unilateral or bilateral cherry-like swelling of the nasal septum that occupies the anterior nasal vestibule to varying degrees; in some instances, posterior nasal septal abscesses can occur. ${ }^{2,9}$ Some would describe the swelling as grayish or bluish. ${ }^{5,14}$ Computed tomography scan with contrast is recommended to confirm abscess size and areas where it may have tracked, especially when complications are suspected or other considerations such as neoplasms are entertained. In this case, it was not done since aspiration pointed toward an abscess formation, and there seemed to be no signs and symptoms of complications. Prompt recognition and treatment are paramount because the associated complications - orbital cellulitis, meningitis, intracranial abscesses, cavernous sinus thrombosis, among others - are life-threatening. ${ }^{5,14}$

Management principles of septal abscesses in patients with chronic kidney disease do not differ from any other patient with a septal abscess. Needle aspiration before incision and drainage can be done to confirm pus and collect specimens for bacteriologic study. Evacuation of the abscess should be carried out with incision and drainage, which can be done under general or local anesthesia, depending on surgeon preference. Broad-spectrum antibiotics may be given intravenously, then switched to oral preparations based on the bacteriologic studies and clinical response. The most common pathogen associated with nasal septal abscess is $S$. aureus. ${ }^{14}$ Some surgeons place a Penrose drain immediately after drainage and a transseptal quilting suture. Nasal packing serves as a stent and prevents reaccumulation of blood and pus. The drain and packs can be maintained for two to three days, then removed on follow-up. Destruction of septal cartilage, which may result in saddle nose deformity, should be reconstructed. Follow-up should be emphasized to the patient to monitor resolution or recurrence.

This case report highlights the possibility of developing nasal infections in patients with end-stage kidney disease due to compromised host defenses. Diagnosis is clinical, and patients most often present with nasal congestion or 
obstruction. Prompt recognition and treatment are vital to avoid life-threatening complications. In those without volume overload, revising the dialysis prescription to a less aggressive regimen is necessary to avoid intradialytic hypotension.

\section{Statement of Authorship}

All authors participated in the data collection and analysis and approved the final version submitted.

\section{Author Disclosure}

All authors declared no conflicts of interest.

\section{Funding Source}

This study was self-funded.

\section{REFERENCES}

1. Gupta KL, Radotra BD, Sakhuja V, Banerjee AK, Chugh, KS. Mucormycosis in Patients with Renal Failure. Renal Failure; 1989; 11(4):195-9.

2. Ambrus PS, Eavey RD, Baker AS, Wilson WR, Kelly JH. Management of Nasal Septal Abscess. Laryngoscope; 1981 Apr; 91(4):575- 82.

3. Mooney CP, Rimmer J. Spontaneous Nasal Septal Hematoma and Abscess: A Case Report and Literature Review. Rhinology Online; 2018 Sept 11; 1:122-6.

4. Nwosu JN, Nnadede PC. Nasal Septal Hematoma/Abscess: Management and Outcome in a Tertiary Hospital of a Developing Country. Patient Prefer Adherence; 2015 Jul 28; 9:1017-1021.
5. Fearon B, McKendry JB, Parker J. Abscess of the Nasal Septum in Children: A Case History of Meningitis Secondary to a Septal Abscess. Arch Otolaryngol; 1961; 74:408-12.

6. Valencia MP, Castillo M. Congenital and Acquired Lesions of the Nasal Septum: A Practical Guide for Differential Diagnosis. RadioGraphics; 2008; 28: 205-23.

7. Fry HJ. The Pathology and Treatment of Haematoma of the Nasal Septum. Br J Plast Surg; 1969; 22(4):331-335.

8. Kumar S, Chakravarti A, Sahni JK, Dubey NK. Ear, Nose, and Throat Manifestations in Pediatric Chronic Renal Failure Patients Undergoing Peritoneal Dialysis. Indian J Otolaryngol Head Neck Surg; 2004; 56(3):205-7.

9. George A, Smith WK, Kumar S, Pfleiderer AG. Posterior Nasal Septal Abscess in a Healthy Adult Patient. J Laryngol Otol; 2008;122(12):1386-8.

10. Shah SB, Murr AH, Lee KC. Nontraumatic Nasal Septal Abscesses in the Immunocompromised: Etiology, Recognition, Treatment, and Sequelae. Am J Rhinol; 2000; 14(1):39-43.

11. Dinesh R, Avatar S, Haron A, Suhana, Azwarizan. Nasal Septal Abscess with Uncontrolled Diabetes Mellitus: Case Reports. Med J Malaysia; 2011; 66(3):253-4.

12. Adler D, Ritz E. Perforation of the Nasal Septum in Patients with Renal Failure. The Laryngoscope; 1980; 90: 317-21.

13. Canty PA, Berkowitz RG. Hematoma and Abscess of the Nasal Septum in Children. Arch Otolaryngol Head Neck Surg; 1996; 122(12):1373-6.

14. Cheng LH, Wu PC, Shih CP, et al. Nasal Septal Abscess: A 10year Retrospective Study. Eur Arch Otorhinolaryngol; 2019; 276(2): 417-20.
Have you read the current trends in Medical and Health Research in the Philippines?

\section{Acta Medica Philippina The National Health Science Journal}

